\title{
Neighborly Maps with Few Vertices
}

\author{
Amos Altshuler ${ }^{1}$ and Ulrich Brehm ${ }^{2}$ \\ ${ }^{1}$ Ben-Gurion University of the Negev, Beer-Sheva, Israel, and \\ Temple University, Philadelphia, Pennsylvania, USA \\ ${ }^{2}$ Technische Universität Berlin, FB Mathematik, Berlin 12, Federal Republic of Germany
}

\begin{abstract}
A neighborly map is a simplicial 2-complex which decomposes a closed 2-manifold without boundary, such that any two vertices are joined by an edge (1-cell) in the complex. We find and describe all the neighborly maps with Euler characteristic $\chi>-10$ (i.e., genus $g<6$, if orientable) or, equivalently, all the neighborly maps with $V<12$ vertices.
\end{abstract}

\section{Introduction}

We consider triangulations of compact closed 2-manifolds without boundary. Such a triangulation is neighborly if any two of its vertices are joined by a (unique) edge. A neighborly triangulation of such a manifold is called also a neighborly map, or neighborly manifold. (Compare [6]).

Neighborly maps play an important role in the generalization of the four-color theorem known as the map color theorem (see [11]). The question of their existence is known in the literature also as "the thread problem" [10, p. 334].

Neighborly maps are also related to the problem of geometrical embedding of simplicial 2-manifolds. The question whether every triangulation is geometrically embeddable in $\mathbb{R}^{3}$ seems to be open for every orientable 2-manifold except the sphere. Orientable neighborly maps of genus $g \geq 6$ are canonical candidates for possible counterexamples and would yield otherwise very. interesting polyhedra. However, for every nonorientable 2-manifold with boundary there exists a triangulation, which does not allow a geometric embedding (even no geometric immersion) in $\mathbb{R}^{3}$ (see [7]).

From Euler's formula $V-E+F=\chi$ and from $V(V-1)=2 E=3 F$ for a neighborly map we get $V^{2}-7 V+6 \chi=0$, thus

$$
V=(7+\sqrt{49-24 \chi}) / 2
$$


where $V, E, F$ denote the number of vertices, edges, and facets, respectively, and $\chi$ denotes the Euler characteristic. As $V \geq 4$ and $\chi \leq 2$ are integers it follows that for $V \equiv 2(\bmod 3)$ neighborly maps cannot exist. Because orientable 2-manifolds have even Euler characteristic the only numbers of vertices which can occur for a neighborly orientable map are of the form $V \equiv 0,3,4$, or $7(\bmod 12)$, (see also [11]). Franklin [9] proved that the Klein bottle does not possess any neighborly triangulation. Ringel, Youngs, and coauthors proved [11] that any other 2manifold of Euler characteristic $\chi$ (whether orientable or not) for which

$$
V=(7+\sqrt{49-24 \chi}) / 2
$$

is an integer, possesses a neighborly triangulation with $V$ vertices.

The first pairs of numbers $(V, \chi)$ satisfying the above equation with $\chi \leq 2$ are $(4,2),(6,1),(7,0),(9,-3),(10,-5),(12,-10)$.

In the present work we describe an algorithm that constructs all the neighborly triangulations of a given 2-manifold. More accurately, given an integer $V$, the algorithm constructs all the neighborly maps, whether orientable or not, with $V$ vertices. However, the amount of computer time increases very fast with $V$, and already for $V=12$ it is practically not applicable (even if restricted to the orientable-or nonorientable-case only). Thus our investigation is confined to $V<12$. The case $V=4$ yields the tetrahedron and is trivial. The cases $V=6,7$ seem to be well known, and they are treated here (in Section 2, and summarized in Theorem 1) mainly as an exemplification of our algorithm. This treatment, however, yields a new proof of Franklin's result concerning the Klein bottle. The 7-vertex triangulation of the torus has been known to Möbius and has been realized as a polyhedron in $\mathbb{R}^{3}$ by Császár. It turns out that the tetrahedron and the Möbius-Császár torus are the only orientable neighborly maps for $\chi<-10$. The algorithm is described in Section 2. In Section 3 it is applied to the cases $V=9,10$. Altogether we find two neighborly maps with 9 vertices $(\chi=-3)$ and 14 neighborly maps with 10 vertices $(\chi=-5)$, all nonorientable. They are described in detail in Tables 1, 2, and 3. Finally, in Section 4, we discuss the automorphism groups of these 16 maps.

\section{The Algorithm}

Let $N$ be a connected simplicial 2-complex with $V$ vertices. Then its body $|N|$ is a 2-manifold and $N$ is a neighborly map on $|N|$ iff:

(a) every edge (1-simplex) in $N$ belongs to precisely two triangles (2-simplices) in $N$;

(b) the link of every vertex in $N$ is a simple circuit of length $V-1$.

If $N$ is such a neighborly map and $D$ is a subcomplex of $N$, we say that $D$ is full if each vertex and edge in $D$ is contained in some triangle in $D$. An edge in $D$ is covered if it belongs to precisely two triangles in $D$. Obviously a full subcomplex $D$ of the neighborly map $N$ satisfies:

$\left(\mathrm{a}^{\prime}\right)$ every edge in $D$ belongs to one or two triangles in $D$; 
$\left(b^{\prime}\right)$ the link of every vertex in $D$ is either a simple circuit of length $V-1$ or a union of disjoint open simple paths.

Also, $D=N$ iff $D$ satisfies (a) or (b) (with $N$ replaced by $D$ ). (If it satisfies one of them then it also satisfies the other.)

For every $V \geq 4$, such that $\chi=\left(7 V-V^{2}\right) / 6$ is an integer, our algorithm is supposed to find all the neighborly maps with $V$ vertices (and Euler characteristic $\chi$ ) whether orientable (for $\chi$ even) or not. The vertices of each such map $N$ are labeled $0,1, \ldots, V-1$, and the (cyclic) order of the vertices in $\operatorname{link}(0, N)$ is 1 , $2, \ldots, V-1$. Thus the simplicial 2-complex $C$ composed of the triangles 012 , $023, \ldots, 0(V-2)(V-1), 0(V-1) 1$ and their faces is a full subcomplex of each such $N$.

The algorithm is a branching process which starts with the complex $D=C$ and adds to it triangles (over the set $\{1,2, \ldots, V-1\}$ ) one at a time, in a corallike manner (compare [2]). Each added triangle covers an existing edge which was uncovered and is added together with its faces. Branching occurs when an edge that is to be covered may be covered in more than one way.

More specifically, in each intermediate step we get a full connected 2-complex $D$. An uncovered edge $e$ in $D$ is chosen, and a triangle $\triangle$ over $\{1,2, \ldots, V-1\}$ that contains $e$ and is not yet in $D$ is taken as a candidate to be added (with its faces) to $D . \triangle$ is a "good" candidate iff $D \cup \triangle$ (more exact, $D \cup\{\delta: \delta \in \triangle\}$ ) satisfies both conditions $\left(a^{\prime}\right)$ and $\left(b^{\prime}\right)$, otherwise it fails. A branching occurs here if there is more than one good candidate to cover the edge $e$. If there is no good candidate at all, it is a failure of the present branch and we return to the previous branching point. A branch ends successfully if in the resulting complex $D$ all the edges are covered, i.e., $D$ satisfies condition (a), therefore also condition (b), and is a neighborly map.

Obviously, a neighborly map with $V$ vertices has $F=V(V-1) / 3$ triangles, thus exactly $V(V-1) / 3-(V-1)=(V-1)(V-3) / 3$ triangles must be added to the starting complex $C$ to yield a neighborly map. The process can be made more efficient by considering the symmetries of $D$ when looking for a candidate to cover an edge in $D$.

We now exemplify this algorithm in the cases $V=6(\chi=1)$ and $V=7(\chi=0)$. We use the symbol $A_{a b}$ to indicate that a triangle $\triangle=a b c$ fails to be a good candidate to cover the edge $a b$ because the edge is in three triangles in $D \cup \triangle$, and condition ( $\left.a^{\prime}\right)$ is therefore violated. $B_{a}$ indicates that $\triangle=a b c$ fails because link $(a, D \cup \triangle)$ contains a circuit of length $<V-1$, and condition $\left(b^{\prime}\right)$ is violated. $C_{a b}$ indicates that a branch ends in a failure because the edge $a b$ in the complex $D$ obtained so far is uncovered, and cannot be covered.

\section{The Case $V=6$}

The triangles in $C$ are $012,023,034,045$, and 051 . Starting with $D=C$ we want to cover 12 . By the symmetry of $C$ we may assume just two candidates: 123 and 124. 123 fails $\left(B_{2}\right)$, so we take $D=C \cup\{124\}$. The candidates to cover 14 are 143 and 145. 145 fails $\left(B_{5}\right)$, so the new $D$ is $C \cup\{124,143\}$. To cover 24,243 fails $\left(A_{34}\right)$ and we take $\triangle=245$. To cover 13 in $D=C \cup\{124,143,245\}$ we cannot use 132 , 
which has already been rejected, and we take $\triangle=135$. Finally, to cover 25 , the only possibility is $\Delta=253$. Now $N=C \cup\{124,134,245,135,235\}$ is indeed a neighborly map with $\chi=1$-a neighborly map on the projective plane-and the only such map.

\section{The Case $V=7$}

Here the triangles in $C$ are $012,023,034,045,056,061$, and eight triangles have to be added to yield a neighborly map either on the torus or on Klein's bottle. The triangles 123 and 126 fail to cover $12\left(B_{2}\right)$ (resp. $\left.B_{1}\right)$, thus the only candidates to cover 12 are 124 and 125 . Because of the symmetry of $C$ we may ignore 125 , and we take $D=C \cup\{124\}$. Now there are three candidates to cover 14:143,145, and 146. 146 fails $\left(B_{1}\right)$. The candidate 143 yields the following branching:

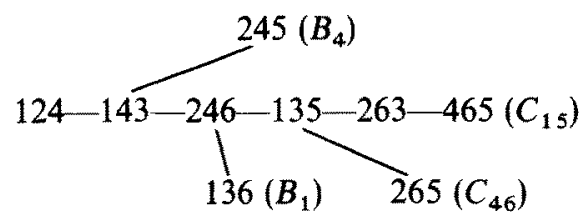

(In most cases we ignore triangles which readily fail to be candidates, and the first two vertices of each candidate $\triangle$ indicate the edge that is to be covered by $\triangle$.) Here, as we see, each branch fails. Finally, the candidate 145 yields the following branching:

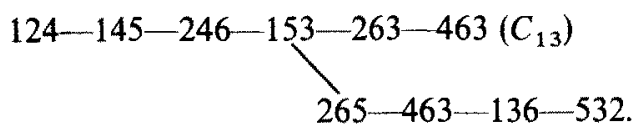

The last branch does not end in a failure. It does indeed yield a neighborly map with 7 vertices and $\chi=0, g=1$. It is readily checked that this map is orientable, and is therefore a torus (see [1, p. 217]). As orientability has not been assumed $a$ priori, it proves that the Klein bottle has no neighborly triangulation. Thus we have proved:

Theorem 1. The projective plane and the torus yield a unique neighborly map each. There is no neighborly map on the Klein bottle.

\section{The Maps with 9 and 10 Vertices}

Using a computer, ${ }^{1}$ we applied this algorithm to 9 vertices $(\chi=-3)$ and we obtained 32 neighborly maps-not necessarily combinatorially distinct. The 32

\footnotetext{
${ }^{1}$ Most of the programming for this section has been done by Yossi Friedman to whom we wish to extend our thanks.
} 
maps have been reduced to just three when the symmetries induced by rotations and reflections of the complex $C=\operatorname{star}(0, N)$ were incorporated in the program. This new version of the program has been applied to 10 vertices $(\chi=-5)$ and after a 14-hour run on Vax 750 it yielded 116 maps.

Two of the three maps with 9 vertices were shown to be isomorphic to each other, and the third is not isomorphic to them. Thus there are exactly two neighborly maps with 9 vertices, and we denote them $N(9,1)$ and $N(9,2)$. They are described in Tables 1 and 2. In Table 1 they are described by means of the list of their triangles, as they originally came out from the computer. (Note that they differ in just four triangles-a fact that is used in [3].) In Table 2 the maps

Table 1. The two neighborly maps with 9 vertices (Euler characteristic $\chi=-3$ ).

\begin{tabular}{|c|c|c|}
\hline \multirow[b]{2}{*}{ Triangles common to $N(9,1), N(9,2)$} & \multicolumn{2}{|c|}{ Additional triangles } \\
\hline & in $N(9,1)$ & in $N(9,2)$ \\
\hline $\begin{array}{lllllll}012 & 023 & 034 & 045 & 056 & 067 & 078\end{array}$ & 146157 & 147156 \\
\hline $\begin{array}{lllllll}018 & 124 & 136 & 137 & 158 & 238 & 245\end{array}$ & 347356 & 346357 \\
\hline $\begin{array}{llllll}257 & 267 & 268 & 358 & 468 & 478\end{array}$ & & \\
\hline
\end{tabular}

Table 2. The two neighborly maps with Euler characteristic $\chi=-3$ given by the links of their vertices and their automorphism groups. $j \cdot a_{1} a_{2} \cdots a_{8}$ means that the link of vertex $j$ is $a_{1} a_{2} \cdots a_{8}$. $x, y$, and $z$ stand for the permutations $x=(02)(31)(76)$, $y=(810)(752)(634), z=(036217)(458)$, respectively.

\begin{tabular}{cccc}
\hline \multirow{2}{*}{ Map $N(9, i)$} & Links & FP-matrix & $\begin{array}{c}\text { Symmetry group, } \\
\text { order, generators }\end{array}$ \\
\hline$i=$ & & & \\
& 0.65432187 & 22425334 & \\
& 1.08573642 & 22425334 & $Z_{6}$ \\
& 2.75410386 & 22425334 & 6 \\
1 & 3.28561740 & 22425334 & \\
& 6.14827053 & 22425334 & \\
& 7.34806251 & 22425334 & \\
& 4.12503786 & 23453524 & \\
& 5.71836042 & 23453524 & \\
& 8.97462351 & 23453524 & \\
& 0.12345678 & 25253344 & \\
& 1.85637420 & 25253344 & \\
& 2.30145768 & 25253344 & \\
2 & 3.85716402 & 25253344 & $S_{3} \times Z_{3}$ \\
& 4.12503687 & 25253344 & 18 \\
& 5.61837240 & 25253344 & $x, y$ \\
& 6.48270513 & 25253344 & \\
& 7.48062531 & 25253344 & \\
& 8.07462351 & 25253344 & \\
\hline
\end{tabular}


are given by the lists of the links of their vertices. The description by the links of the vertices, rather than by the list of triangles, simplifies the investigation of the automorphism group of the maps.

To check the 116 maps with 10 vertices for isomorphism is of course much more complicated. For this purpose, we use the fingerprint-matrix which has been introduced in [3]. Here we recall the definition.

Let $N$ be a neighborly map with $V$ vertices and $x$ a vertex of $N$. Let $k:=V-1$ and $y_{1} y_{2}, \ldots, y_{k}$ be the link of $x$ (i.e., $x y_{i-1} y_{i}$ are triangles of the map for $i=1, \ldots, k$ where $y_{0}:=y_{k}$ ). Let $y_{i-1} y_{i} z_{i}$ with $z_{i} \neq x$ be a triangle of $N$. Then there is a unique number $\varphi(i)$ with $2 \leq \varphi(i) \leq V-4$ with $z_{i}=y_{i+\varphi(i)}$ or with $z_{i}=y_{i+\phi(i)-k}$, because $N$ is neighborly.

Now we define the fingerprint-vector (FP-vector) of $x$ to be the first (smallest) tuple among

$$
\begin{gathered}
\{(\varphi(i), \varphi(i+1), \ldots, \varphi(k), \varphi(1), \ldots, \varphi(i-1)) \mid i=1, \ldots, k\} \\
\cup\{\overline{\varphi(i)}, \overline{\varphi(i-1)}, \ldots, \overline{\varphi(1)}, \overline{\varphi(k)}, \ldots, \overline{\varphi(i+1)} \mid i=1, \ldots, k\}
\end{gathered}
$$

where $\overline{\varphi(i)}:=V-2-\varphi(i)$ with respect to the lexicographic ordering (of tuples of natural numbers). From the definition follows immediately that the FP-vector of a vertex does not depend on the labeling of the vertices nor on the chosen representation of the link.

Now the fingerprint-matrix (FP-matrix) $\mathrm{FP}(N)$ of $N$ is the $V \times(V-1)$-matrix in which the rows are the FP-vectors ordered lexicographically (the first row is the lexicographically smallest FP-vector and so on). Thus by construction two neighborly maps which are isomorphic have identical FP-matrices. Conversely, in all our examples of neighborly maps, two neighborly maps with the same FP-matrix are isomorphic. Whether this holds in general is an interesting open problem.

For each of the 116 maps under consideration, the FP-matrix has been calculated. It turned out that the 116 maps split into 14 equivalence classes with respect to the FP-matrices, and that the maps within each equivalence class are isomorphic to each other. Thus there are precisely 14 distinct neighborly 2manifolds with 10 vertices. We denote them by $N(10, i), 1 \leq i \leq 14$, the order being according to the lexicographic order of the FP-matrices. These 14 maps are described in Table 3 by means of the links of their vertices, and their FP-matrices are given.

We have thus completed the proof of

Theorem 2. There are precisely two neighborly 2-manifolds with 9 vertices $(\chi=-3)$ and precisely 14 neighborly 2 -manifolds with 10 vertices $(\chi=-5)$. They are listed in Tables 2 and 3. 
Table 3. The 14 neighborly maps with Euler characteristic $\chi=-5$ given by the links of their vertices. Legend for permutations: $a=(01)(23)(45)(67), b=(012)(345)(678), c=(01)(23)(67)(89)$,

$d=(012345678), e=$ identity, $f=(01234)(56789)$,

$g=(01)(29)(37)(45), h=(12)(38)(47)(56), j=(01)(23)(45)(78)$,

$k=(012)(469)(578)$.

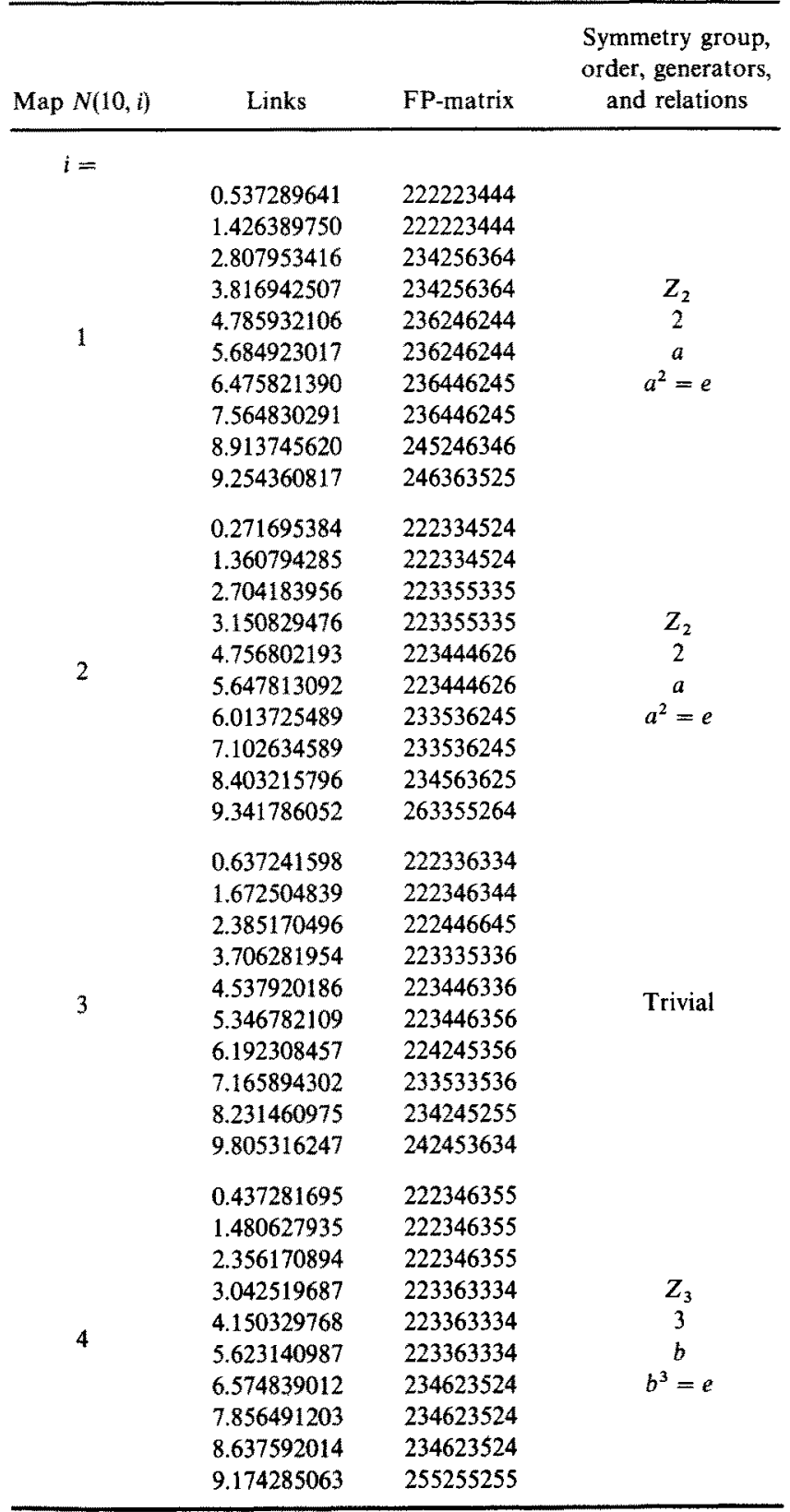




\begin{tabular}{|c|c|c|c|}
\hline $\operatorname{Map} N(10, i)$ & Links & FP-matrix & $\begin{array}{l}\text { Symmetry group, } \\
\text { order, generators, } \\
\text { and relations }\end{array}$ \\
\hline \multirow{10}{*}{5} & 0.824159673 & 222426445 & \multirow{10}{*}{$\begin{array}{c}Z_{2} \\
2 \\
j \\
j^{2}=e\end{array}$} \\
\hline & 1.350496827 & 222426445 & \\
\hline & 2.179365408 & 235236244 & \\
\hline & 3.892645170 & 235236244 & \\
\hline & 4.635201987 & 236363334 & \\
\hline & 5.243109786 & 236363334 & \\
\hline & 6.185234709 & 242464635 & \\
\hline & 7.603129584 & 245336426 & \\
\hline & 8.561203947 & 245336426 & \\
\hline & 9,506148327 & 262634445 & \\
\hline \multirow{10}{*}{6} & 0.653871294 & 222444625 & \multirow{10}{*}{ Trivial } \\
\hline & 1.025983467 & 223344224 & \\
\hline & 2.109763485 & 224256355 & \\
\hline & 3.805796241 & 234246264 & \\
\hline & 4.287590613 & 235346426 & \\
\hline & 5.128603749 & 235536245 & \\
\hline & 6.327140589 & 236253334 & \\
\hline & 7.392610845 & 236363425 & \\
\hline & 8.703196524 & 245264536 & \\
\hline & 9.736815402 & 246263345 & \\
\hline \multirow{10}{*}{7} & 0.378195426 & 222446645 & \multirow{10}{*}{$\begin{array}{c}Z_{2} \\
2\end{array}$} \\
\hline & 1.690854372 & 222446645 & \\
\hline & 2.857160493 & 225334426 & \\
\hline & 3.560714829 & 225334426 & \\
\hline & 4.513867920 & 234246456 & \\
\hline & 5.814093672 & 235636425 & \\
\hline & 6.847530219 & 236346446 & \\
\hline & 7.894652130 & 236346446 & \\
\hline & 8.469701523 & 236463535 & \\
\hline & 9.478610532 & 236463535 & \\
\hline \multirow{10}{*}{8} & 0.498576321 & 223364634 & \multirow{10}{*}{ Trivial } \\
\hline & 1.479356820 & 224224424 & \\
\hline & 2.184695730 & 225236244 & \\
\hline & 3.602748519 & 225336424 & \\
\hline & 4.738265901 & 235236446 & \\
\hline & 5.492708316 & 236345336 & \\
\hline & 6.245187039 & 236453524 & \\
\hline & 7.250689143 & 242526245 & \\
\hline & 8.216790534 & 244263634 & \\
\hline & 9.625408713 & 246363534 & \\
\hline \multirow{10}{*}{9} & 0.914275638 & 223423524 & \multirow{10}{*}{$\begin{array}{c}Z_{9} \\
9 \\
d \\
d^{9}=e\end{array}$} \\
\hline & 1.253867409 & 223423524 & \\
\hline & 2.364078519 & 223423524 & \\
\hline & 3.947518062 & 223423524 & \\
\hline & 4.958620173 & 223423524 & \\
\hline & 5.960731284 & 223423524 & \\
\hline & 6.971842305 & 223423524 & \\
\hline & 7.820534169 & 223423524 & \\
\hline & 8.903164527 & 223423524 & \\
\hline & 9.801234567 & 333333335 & \\
\hline
\end{tabular}




\begin{tabular}{|c|c|c|c|}
\hline $\operatorname{Map} N(10, i)$ & Links & FP-matrix & $\begin{array}{l}\text { Symmetry group, } \\
\text { order, generators, } \\
\text { and relations }\end{array}$ \\
\hline \multirow{10}{*}{10} & 0.961752483 & 224224224 & \multirow{10}{*}{$\begin{array}{c}A_{4} \\
12 \\
c, k \\
k^{3}=c^{2}=(c k)^{3}=e\end{array}$} \\
\hline & 1.928706534 & 224224224 & \\
\hline & 2.405819736 & 224224224 & \\
\hline & 3.908627415 & 224224224 & \\
\hline & 4.026573198 & 233556345 & \\
\hline & 5.316470289 & 233556345 & \\
\hline & 6.238790154 & 233556345 & \\
\hline & 7.329681054 & 233556345 & \\
\hline & 8.304952176 & 233556345 & \\
\hline & 9.035841276 & 233556345 & \\
\hline \multirow{10}{*}{11} & 0.364528719 & 224425525 & \multirow{10}{*}{ Trivial } \\
\hline & 1.907386542 & 225236345 & \\
\hline & 2.341968057 & 225346234 & \\
\hline & 3.271856094 & 234236244 & \\
\hline & 4.239876051 & 234426426 & \\
\hline & 5.836140279 & 236263334 & \\
\hline & 6.153047928 & 236363424 & \\
\hline & 7.310846952 & 245262534 & \\
\hline & 8.613594702 & 246263344 & \\
\hline & 9.576210348 & 255355526 & \\
\hline \multirow{10}{*}{12} & 0.637492815 & 234425526 & \multirow{10}{*}{$Z_{5}$} \\
\hline & 1.480539267 & 234425526 & \\
\hline & 2.809164537 & 234425526 & \\
\hline & 3.152706489 & 234425526 & \\
\hline & 4.526381709 & 234425526 & \\
\hline & 5.879423106 & 236445526 & \\
\hline & 6.985034217 & 236445526 & \\
\hline & 7.859614032 & 236445526 & \\
\hline & 8.965720143 & 236445526 & \\
\hline & 9.576831204 & 236445526 & \\
\hline \multirow{10}{*}{13} & 0.162937458 & 246246246 & \multirow{10}{*}{$\begin{array}{c}A_{\mathrm{s}} \\
60 \\
f, g \\
f^{5}=g^{2}=(f g)^{3}=e\end{array}$} \\
\hline & 1.537296084 & 246246246 & \\
\hline & 2.357190648 & 246246246 & \\
\hline & 3.251709468 & 246246246 & \\
\hline & 4.826397051 & 246246246 & \\
\hline & 5.896723140 & 246246246 & \\
\hline & 6.201957834 & 246246246 & \\
\hline & 7.986521304 & 246246246 & \\
\hline & 8.597632410 & 246246246 & \\
\hline & 9.021658743 & 246246246 & \\
\hline \multirow{10}{*}{14} & 0.782561349 & 253453626 & \multirow{10}{*}{$\begin{array}{c}6 \\
b, h \\
b^{3}=h^{2}=(b h)^{2}=e\end{array}$} \\
\hline & 1.860372459 & 253453626 & \\
\hline & 2.671480539 & 253453626 & \\
\hline & 3.104685297 & 2.55534526 & \\
\hline & 4.215763098 & 255534526 & \\
\hline & 5.238741960 & 255534526 & \\
\hline & 6.018347295 & 255534526 & \\
\hline & 7.126458093 & 255534526 & \\
\hline & 8.207536194 & 255534526 & \\
\hline & 9.704815623 & 264264264 & \\
\hline
\end{tabular}




\section{Automorphism Groups}

The automorphism groups of our 16 neighborly 2 -manifolds with 9 and 10 vertices have been checked both by hand and by computer. The results are as follows:

\section{Vertices}

$N(9,1)$. The automorphism group is $Z_{6}$. It is generated by the permutation $z=\left(\begin{array}{llll}0 & 362 & 17\end{array}\right)(458)$. There are four orbits of triangles each of length 6 generated by the triangles $012,034,045,056$.

$N(9,2)$. The automorphism group is $S_{3} \times Z_{3}$. It is of order 18 and acts as follows:

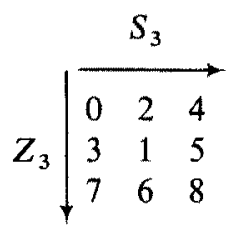

The rows can be permutated cyclically and the columns can be permutated arbitrarily. Generators of this group are

$$
x:=\left(\begin{array}{lll}
0 & 2
\end{array}\right)(31)(76)
$$

and

$$
y:=\left(\begin{array}{lll}
8 & 1 & 0
\end{array}\right)\left(\begin{array}{lll}
7 & 5 & 2
\end{array}\right)\left(\begin{array}{lll}
6 & 3 & 4
\end{array}\right)
$$

Note that

$$
\begin{aligned}
& u:=\left(\begin{array}{llll}
0 & 2 & 4
\end{array}\right)\left(\begin{array}{lll}
3 & 1 & 5
\end{array}\right)(768)=y x y^{-1} x, \\
& v:=\left(\begin{array}{lll}
0 & 3 & 7
\end{array}\right)(216)(458)=x y x y, \\
& y=v^{-1} u^{-1},
\end{aligned}
$$

showing that the permutation group $S_{3} \times Z_{3}$, generated by $x, u, v$, can be generated also by $x$ and $y$. After a suitable relabeling of the vertices we get the automorphism group of $N(9,1)$ as a subgroup of the automorphism group of $N(9,2)$, because $x y=\left(\begin{array}{lllll}0 & 8 & 34 & 4 & 5\end{array}\right)\left(\begin{array}{ll}6 & 12\end{array}\right)$ is conjugate to $z$ in $S_{9}$.

\section{Vertices}

Each of the maps $N(10,3), N(10,6), N(10,8)$, and $N(10,11)$ has the trivial group as its symmetry group. (Note that in each of the corresponding FP-matrices no two rows are identical.) 
We use the following notation for some permutations over the set $\{0,1, \ldots, 9\}$ :

$$
\begin{array}{ll}
a=(01)(23)(45)(67), & f=(01234)(56789), \\
b=(012)(345)(678), & g=(01)(29)(37)(45), \\
c=(01)(23)(67)(89), & h=(12)(38)(47)(56), \\
d=(012345678), & j=(01)(23)(45)(78), \\
e=\text { identity, } & k=(012)(469)(578) .
\end{array}
$$

In each of the maps $N(10,1), N(10,2), N(10,5)$, and $N(10,7)$, the group $Z_{2}$ (the cyclic group of order 2) generated by $a$ acts as the symmetry group, with the vertices 8 and 9 fixed.

In $N(10,4)$ the symmetry group is $Z_{3}$, generated by $b$, and the vertex 9 is a fixed point.

$N(10,9)$ has $Z_{9}$, the cyclic group of order 9 , as its symmetry group, with $d$ as generator and 9 as fixed point.

The group of $N(10,10)$ is of order 12 . It is the alternating group $A_{4}$ with generators $b, c$, and relations $c^{2}=b^{3}=(b c)^{3}=e(b c$ reads: first $c$ and then $b)$. This follows from the fact that the group must preserve the set of vertices $\{0,1,2,9\}$ (as read from the FP-matrix), it is transitive on the ordered pairs from this set, and any correspondence $(i, j) \rightarrow(k, l)$ of such ordered pairs determines a unique automorphism of the map. Note that $N(10,10)$ is closely related to a certain polyhedron of genus 3 having the same automorphism group. More precisely, when replacing in $N(10,10)$ the six triangles $456,457,678,679,849$, and 895 by the four triangles $469,478,568$, and 579 we get a triangulation of an orientable manifold of genus 3 having the same automorphism group. This triangulated 2-manifold can be realized as a polyhedron of genus 3 in $\mathbb{R}^{3}$ (without selfintersections) as has been shown in [5].

The relabeling of the vertices of the orientable 2 -manifold of genus 3 derived from $N(10,10)$ to the vertices of the polyhedron described in [5] is given by the permutation $(0123457)(698)$.

The group of $N(10,12)$ is of order 5 . It is the cyclic group $Z_{5}$ with $f$ as generator and with no fixed point.

Most interesting is the group of $N(10,13)$. On one hand, it is of order $\leq 60$. (It follows from the FP-matrix that for each two vertices $i, j$ there are at most six automorphisms mapping $i$ on $j$.) On the other hand, the group has the generators $f, g$ with the relations $f^{5}=g^{2}=(f g)^{3}=e$, and these define the alternating group $A_{5}$ (known also as the icosahedral group) which is of order 60 . Thus the symmetry group of $N(10,13)$ is $A_{5}$. It operates transitively on the vertices and on the triangles.

It is worth noting that $N(10,13)$ can be obtained from the hemidodecahedron (the dodecahedron with antipodal points being indentified) by replacing each of the six pentagons by the unique triangulated Möbius strip with 5 vertices and with the same boundary as the pentagon. This type of construction has also been described in [4]. More geometrically, we can get $N(10,13)$ from the regular dodecahedron by replacing each pair of opposite pentagons by the ten triangles on the boundary of the convex hull of the union of the two pentagons. This yields 
a starpolyhedron of genus 6 . Finally, we get $N(10,13)$ by identifying pairs of points with respect to the center of symmetry. Note that the (geometric) symmetry group of the starpolyhedron operates transitively on the vertices and on the faces.

Finally, the symmetry group of $N(10,14)$ is of order 6 . It is the symmetric group $S_{3}$, generated by $b, h$ with the relations $h^{2}=b^{3}=(b h)^{2}=e$ and with 9 as fixed point.

All this information about the symmetry groups of the neighborly maps with 9 and 10 vertices is summarized in Tables 2 and 3 .

\section{References}

1. A. Altshuler, Polyhedral realization in $\mathbb{R}^{3}$ of triangulations of the torus and 2-manifolds in cyclic 4-polytopes. Discrete Math. 1 (1971), 211-238.

2. A. Altshuler, Combinatorial 3-manifolds with few vertices. J. Combin. Theory 16(1974), 165-173.

3. A. Altshuler, On neighborly triangulations of 2-manifolds. (In preparation.)

4. A. Altshuler and U. Brehm, The weakly neighborly polyhedral maps on the 2-manifold with Euler Characteristic - 1, Discrete Comput. Geom. 1 (1986), 355-369.

5. J. Bokowski and U. Brehm, A New Polyhedron of Genus 3 with 10 Vertices, Colloquia Mathematica Societatis János Bolyai 48. Intuitive Geometry, Siófok, North-Holland, Amsterdam, 1985, pp. $105-116$.

6. U. Brehm and A. Altshuler, On weakly neighborly polyhedral maps of arbitrary genus. Israel J. Math. 53 (1986), 137-157.

7. U. Brehm, A nonpolyhedral triangulated Möbius strip. Proc. Amer. Math. Soc. 89(1983), 519-522.

8. H. S. M. Coxeter and W. O. J, Moser, Generators and Relations for Discrete Groups. SpringerVerlag, New York, 1980).

9. P. Franklin, A six color problem, J. Math. Phys. 13 (1934), 363-369.

10. D. Hilbert and S. Cohn-'ossen, Geometry and the Imagination, Chelsea, New York, 1956.

11. G. Ringel, Map Color Theorem, Springer-Verlag, New York, 1974.

Received August 15, 1990, and in revised form June 13,1991. 